\title{
A contribution to the lichen flora of Melville Bugt north of lat. $75^{\circ} \mathrm{N}$, North West Greenland
}

\author{
Hansen, Eric Steen
}

Published in:

Botanica Lithuanica

DOI:

10.2478/v10279-012-0020-8

Publication date:

2012

Document version

Publisher's PDF, also known as Version of record

Document license:

CC BY-NC-ND

Citation for published version (APA):

Hansen, E. S. (2012). A contribution to the lichen flora of Melville Bugt north of lat. $75^{\circ} \mathrm{N}$, North West Greenland. Botanica Lithuanica, 18(2), 117-122. https://doi.org/10.2478/v10279-012-0020-8 


\title{
A CONTRIBUTION TO THE LICHEN FLORA OF MELVILLE BUGT NORTH OF LAT. $7^{\circ} \mathrm{N}$, NORTH WEST GREENLAND
}

\author{
Eric Steen Hansen
}

Natural History Museum of Denmark of Copenhagen University, Botanical Museum, Gothersgade 130, DK-1123 Copenhagen, Denmark; e-mail: erich@snm.ku.dk

\begin{abstract}
Hansen E. S., 2012: A contribution to the lichen flora of Melville Bugt north of lat. $75^{\circ} \mathrm{N}$, North West Greenland [Papildomi duomenys apie Melville Bugt (šiaurės vakarinè Grenlandija) kerpių florą šiauriau $75^{\circ} \mathrm{N}$ platumos]. - Bot. Lith., 18(2): 117-122.

The paper lists 59 species of lichens from Melville Bugt north of $75^{\circ} \mathrm{N}$. Of these, 54 species were recorded for the first time. The lichen vegetation consists of species found in more or less wind-exposed fell fields, snow patches and on rocks.
\end{abstract}

Keywords: lichens, Melville Bugt, Greenland.

\section{INTRODUCTION}

Melville Bugt is situated between Upernavik at c. $73^{\circ} \mathrm{N}$ and the Kap York Peninsula at c. $76^{\circ} \mathrm{N}$ in North West Greenland. Larger stretches of ice-free land do not occur in this part of Greenland, as the inland ice extends to the sea in most places apart from a number of peninsulas and semi-nunataks, which break the ice margin (SørENSEN, 1943). Numerous ice-free islands and skerries occur along the coast. Melville Bugt was practically unknown lichenologically until about thirty years ago. Some lichens from the region have been mapped by Thomson $(1984,1997)$. The Swedish botanist collected a few lichens, for example, Flavocetraria cucullata and Melanelia hepatizon, on Tasiussaq Island in 1916 just before the Second Thule Expedition. The Danish botanists C. Bay and B. Fredskild collected phanerogams, mosses and lichens from different localities in Melville Bugt as the members of the Knud Rasmussen Memorial Expedition in summer 1979 (FredskiLd et al., 1979). The lichen material was handed over to the author for identification. A total of 59 species of lichens collected during the expedition have been identified and form the basis of the present paper. In 1989, the author collected 167 species of lichens in Upernavik Ø just south of Melville Bugt (HANSEN, 2007). In 2009, 172 species of lichens were collected in Qaanaaq and Siorapaluk north of Melville Bugt (Hansen, 2011). Saxicolous lichens are poorly represented in C. Bay's and B. Fredskild's collections. No doubt the future visitors with particular interest in lichens will be able to considerably increase the number of known lichen species from this inhospitable region.

\section{MATERIALS AND METHODS}

The present paper is based on the collections made up by C. Bay and B. Fredskild in July and August 1979. Lichens were collected from numerous localities on the c. $20 \mathrm{~km}$ long peninsula Tugtuligssuaq and four islands south and west of Tugtuligssuaq, viz. Red Head, Depot Øer, N. E. Balle Ø and Sabine Øer (the coordinates of the localities are given in the list of species). The collected material, a total of 90 lichen specimens, was studied using Zeiss light microscopes and identified by the author. The no- 
menclature in the list follows SANTESSON et al. (2004) when appropriate.

The specimens are deposited at the Botanical $\mathrm{Mu}-$ seum of the University of Copenhagen (C).

\section{CHARACTERISTICS OF THE STUDY AREA}

The bedrock in the investigation area is mainly composed of Archaean gneiss and other siliceous rocks. Locally these rocks are intruded by younger basaltic rocks (SøRENSEN, 1943). The mountains rise to altitudes of about $1000 \mathrm{~m}$ a. s. 1 . All rock samples were acid.

Floristically and climatically, the localities are situated in the medium arctic, oceanic region (JENSEN, 1999). However, Melville Bugt is the narrowest and probably also the youngest of the coastal stretches around the Greenland ice shield and, therefore, poor in phanerogams. C. Bay's and B. Fredskild's collecting efforts on Tugtuligssuaq during 40 days resulted in 83 species only (FREDSKILD et al., 1979). Cassiope tetragona and Salix arctica are the dominant dwarf shrub species found growing in snow patches on the lowland. Mixed dwarf shrub heaths composed of $\mathrm{Vac}$ cinium uliginosum, Empetrum hermaphroditum and Salix arctica occur in many places, but they are usually fragmentary. They prefer stable soil just as the scattered populations of Dryas integrifolia. Lichens occur abundantly in these communities as well as on rocks and boulders on talus slopes and in block fields. The climate of Melville Bugt is comparable with that of Upernavik. According to the measurements made by Asiaq/Grønlands Forundersøgelser, the mean temperature in July is $5{ }^{\circ} \mathrm{C}$ in Upernavik, while the mean temperature in March is $-20^{\circ} \mathrm{C}$. The annual precipitation in Upernavik is $>370 \mathrm{~mm}$ (2000).

\section{RESULTS AND DISCUSSION}

\section{List of species}

An asterisk (*) in front of the name in the list of lichens indicates that the taxon is an addition to the lichen flora of Melville Bugt north of $75^{\circ} \mathrm{N}$. CB = Christian Bay; BF $=$ Bent Fredskild.

* Alectoria ochroleuca (Hoffm.) A. Massal. Summit of Red Head, $75^{\circ} 04^{\prime} \mathrm{N}, 58^{\circ} 06^{\prime} \mathrm{W}$, alt. c. 260 $\mathrm{m}$, on soil rich in humus together with Cetraria islandica, C. muricata, Flavocetraria nivalis and Thamnolia vermicularis v. subuliformis, BF, 14 July 1979; Depot Øer, the easternmost island, $75^{\circ} 07^{\prime} \mathrm{N}, 58^{\circ} 33^{\prime} \mathrm{W}$, on soil together with Bryoria nitidula, Flavocetraria nivalis, Ochrolechia frigida and Peltigera malacea, BF, 14 July 1979; N. E. Balle Ø, $75^{\circ} 12^{\prime} \mathrm{N}, 58^{\circ} 54^{\prime} \mathrm{W}$, on soil rich in humus together with, for example, Bryoria nitidula, Cetraria muricata, C. nigricans, Cladonia mitis, C. trassii and Flavocetraria nivalis, BF, 14 July 1979; Tugtuligssuaq, point $619,75^{\circ} 24^{\prime} \mathrm{N}, 58^{\circ} 31^{\prime} \mathrm{W}$, alt. $619 \mathrm{~m}$, on soil on SE slope together with Bryoria nitidula, B. chalybeiformis, Cladonia pyxidata and Stereocaulon glareosum, CB \& BF, 22 July 1979; Tugtuligssuaq, $75^{\circ} 24^{\prime} \mathrm{N}, 58^{\circ} 33^{\prime} \mathrm{W}$, alt. $350 \mathrm{~m}$, on soil on S slope together with Bryocaulon divergens, Bryoria chalybeiformis, Flavocetraria nivalis, Gowardia nigricans and Rinodina mniaraea, CB \& BF, 14 July 1979; Tugtuligssuaq, point $863,75^{\circ} 27^{\prime} \mathrm{N}, 38^{\circ} 11^{\prime} \mathrm{W}$, alt. $860 \mathrm{~m}$, on soil together with Bryoria chalybeiformis, Solorina crocea, Sphaerophorus fragilis and Stereocaulon glareosum, BF, 7 August 1979.

Arctocetraria nigricascens (Nyl.) Kärnefelt \& Thell - Tugtuligssuaq, area southwest of point 863 , $75^{\circ} 26^{\prime} \mathrm{N}, 58^{\circ} 12^{\prime} \mathrm{W}$, alt. $720 \mathrm{~m}$, on moist soil in $\mathrm{S}$ snow patch together with Cetraria islandica, Cladonia macroceras, Dactylina ramulosa, Parmelia skultii, Solorina crocea and the moss, Anthelia juratzkana, CB \& BF, 7 August 1979. A. nigricascens was previously reported from Melville Bugt by the author (HANSEN, 1981). In Greenland, this amphiberingian lichen is restricted to the coastal region north of Disko Bugt in West Greenland and Scoresby Sund in East Greenland (HANSEN, 1995). It is also known from North America, Siberia and Svalbard (Øvstedal et al., 2009; Kristinsson et al., 2010).

* Brodoa oroarctica (Krog) Goward - Tugtuligssuaq, Igdluminerssuit, $75^{\circ} 22^{\prime} \mathrm{N}, 58^{\circ} 32^{\prime} \mathrm{W}$, alt. 20 $\mathrm{m}$, on siliceous stone together with Melanelia hepatizon, Ophioparma lapponica and Pseudephebe minuscula, CB \& BF, 24 July 1979.

* Bryocaulon divergens (Ach.) Kärnefelt - Tugtuligssuaq, Igdluminerssuit, $75^{\circ} 22^{\prime} \mathrm{N}, 58^{\circ} 35^{\prime} \mathrm{W}$, alt. 25 m, on soil, CB \& BF, 25 July 1979; Tugtuligssuaq, $75^{\circ} 24^{\prime} \mathrm{N}, 58^{\circ} 33^{\prime} \mathrm{W}$, alt. $350 \mathrm{~m}$, on dry soil in S fell field, CB \& BF, 22 July 1979; Tugtuligssuaq, area between Itivdlip aluk and point $480,75^{\circ} 26^{\prime} \mathrm{N}, 58^{\circ} 22^{\prime} \mathrm{W}$, alt. $350 \mathrm{~m}$, on soil together with Flavocetraria nivalis, 
CB \& BF, 8 August 1979; Sabine Øer, the biggest island, $75^{\circ} 30^{\prime} \mathrm{N}, 60^{\circ} 13^{\prime} \mathrm{W}$, on soil, BF, 14 July 1979.

* Bryoria chalybeiformis (L.) Brodo \& D. Hawksw. - Tugtuligssuaq, area south of point 419, $75^{\circ} 22^{\prime} \mathrm{N}, 58^{\circ} 35^{\prime} \mathrm{W}$, alt. $250 \mathrm{~m}$, on soil on talus slope together with Luzula confusa, CB \& BF, 18 July 1979; Tugtuligssuaq, point $619,75^{\circ} 24^{\prime} \mathrm{N}, 58^{\circ} 31^{\prime} \mathrm{W}$, alt. $619 \mathrm{~m}$, on soil on SE slope, CB \& BF, 22 July 1979; Tugtuligssuaq, $75^{\circ} 24^{\prime} \mathrm{N}, 58^{\circ} 33^{\prime} \mathrm{W}$, alt. $350 \mathrm{~m}$, on dry soil in fell field, CB \& BF, 22 July 1979; Tugtuligssuaq, point $863,75^{\circ} 27^{\prime} \mathrm{N}, 58^{\circ} 11^{\prime} \mathrm{W}$, alt. $860 \mathrm{~m}$, on soil, BF, 7 August 1979.

* Bryoria nitidula (Th. Fr.) Brodo \& D. Hawksw. - Depot Øer, the easternmost island, $75^{\circ} 07^{\prime} \mathrm{N}, 58^{\circ} 33^{\prime} \mathrm{W}$, on soil, BF, 14 July 1979; N. E. Balle $\varnothing, 75^{\circ} 12^{\prime} \mathrm{N}, 58^{\circ} 54^{\prime} \mathrm{W}$, on soil, BF, 14 July 1979; Tugtuligssuaq, point $619,75^{\circ} 24^{\prime} \mathrm{N}, 58^{\circ} 31^{\prime} \mathrm{W}$, alt. 619 m, on soil, CB \& BF, 22 July 1979.

* Caloplaca nivalis (Körb.) Th. Fr. - Tugtuligssuaq, c. $75^{\circ} 23^{\prime} \mathrm{N}, 58^{\circ} 31^{\prime} \mathrm{W}$, on moss, $\mathrm{CB} \& \mathrm{BF}$, 2 August 1979.

* Candelariella aurella (Hoffm.) Zahlbr. - Tugtuligssuaq, Kap Seddon, $75^{\circ} 22^{\prime} \mathrm{N}, 58^{\circ} 39^{\prime} \mathrm{W}$, on old bone together with Lecanora hagenii v. fallax, CB \& BF, 20 July 1979.

* Candelariella canadensis H. Magn. - Tugtuligssuaq, c. $75^{\circ} 23^{\prime} \mathrm{N}, 58^{\circ} 31^{\prime} \mathrm{W}$, on mineral soil, $\mathrm{CB}$ \& BF, 2 August 1979.

* Cetraria islandica (L.) Ach. - Summit of Red Head, $75^{\circ} 04^{\prime} \mathrm{N}, 58^{\circ} 06^{\prime} \mathrm{W}$, alt. $260 \mathrm{~m}$, on soil rich in humus, BF, 14 July 1979; N. E. Balle Ø, $75^{\circ} 12^{\prime} \mathrm{N}$, $58^{\circ} 54^{\prime} \mathrm{W}$, on soil, BF, 14 August 1979; Tugtuligssuaq, $75^{\circ} 23^{\prime} \mathrm{N}, 58^{\circ} 31^{\prime} \mathrm{W}$, alt. $100 \mathrm{~m}$, on soil, CB \& BF, 14 August 1979; Tugtuligssuaq, southwest of point 863 , $75^{\circ} 26^{\prime} \mathrm{N}, 58^{\circ} 12^{\prime} \mathrm{W}$, alt. $720 \mathrm{~m}$, on soil in snow patch, CB \& BF, 7 August 1979; Sabine Øer, the biggest island, $75^{\circ} 30^{\prime} \mathrm{N}, 60^{\circ} 13^{\prime} \mathrm{W}$, on soil, BF, 14 July 1979.

* Cetraria muricata (Ach.) Eckfeldt - Summit of Red Head, $75^{\circ} 04^{\prime} \mathrm{N}, 58^{\circ} 06^{\prime} \mathrm{W}$, alt. $260 \mathrm{~m}$, on soil rich in humus, BF, 14 July $1979 ;$ N. E. Balle Ø, $75^{\circ} 12^{\prime} \mathrm{N}$, 5854’ W, on soil, BF, 14 August 1979.

* Cetraria nigricans Nyl. - N. E. Balle Ø, $75^{\circ} 12^{\prime} \mathrm{N}, 58^{\circ} 54^{\prime} \mathrm{W}$, on soil, BF, 14 August 1979; Tugtuligssuaq, point $619,75^{\circ} 24^{\prime} \mathrm{N}, 58^{\circ} 31^{\prime} \mathrm{W}$, alt. 619 m, on soil, CB \& BF, 22 July 1979.

* Cetrariella delisei (Bory ex Schaer.) Kärnefelt $\&$ Thell - N. E. Balle Ø, $75^{\circ} 12^{\prime} \mathrm{N}, 58^{\circ} 54^{\prime} \mathrm{W}$, on soil, BF, 14 August 1979.
* Cladonia amaurocraea (Flörke) Schaer. - Tugtuligssuaq, Igdluminerssuit, $75^{\circ} 22^{\prime} \mathrm{N}, 58^{\circ} 35^{\prime} \mathrm{W}$, alt. $25 \mathrm{~m}$, on soil, CB \& BF, 25 July 1979; Tugtuligssuaq, point $619,75^{\circ} 24^{\prime} \mathrm{N}, 58^{\circ} 31^{\prime} \mathrm{W}$, alt. $619 \mathrm{~m}$, on soil, $\mathrm{CB}$ \& BF, 22 July 1979.

* Cladonia arbuscula (Wallr.) Flot. ssp. mitis (Sandst.) Ruoss - N. E. Balle Ø, $75^{\circ} 12^{\prime} \mathrm{N}, 58^{\circ} 35^{\prime} \mathrm{W}$, on soil, BF, 14 August 1979.

* Cladonia bellidiflora (Ach.) Schaer. - N. E. Balle $\varnothing, 75^{\circ} 12^{\prime} \mathrm{N}, 58^{\circ} 54^{\prime} \mathrm{W}$, on soil together with Pertusaria oculata, BF, 14 August 1979; Tugtuligssuaq, Igdluminerssuit, $75^{\circ} 22^{\prime} \mathrm{N}, 58^{\circ} 37^{\prime} \mathrm{W}$, alt. 25 $\mathrm{m}$, on soil together with Flavocetraria nivalis, $\mathrm{CB}$ \& BF, 23 July 1979; Sabine Øer, the biggest island, $75^{\circ} 30^{\prime} \mathrm{N}, 60^{\circ} 13^{\prime} \mathrm{W}$, on soil together with Cladonia borealis, BF, 14 July 1979.

* Cladonia borealis S. Stenroos - Tugtuligssuaq, c. $75^{\circ} 23^{\prime} \mathrm{N}, 58^{\circ} 31^{\prime} \mathrm{W}$, on soil, CB \& BF, 2 August 1979; Sabine Øer, the biggest island, $75^{\circ} 30^{\prime} \mathrm{N}$, $60^{\circ} 13^{\prime} \mathrm{W}$, on soil, BF, 14 July 1979.

* Cladonia chlorophaea (Flörke ex Sommerf.) Spreng. s. lato-Tugtuligssuaq, c. $75^{\circ} 23^{\prime} \mathrm{N}, 58^{\circ} 31^{\prime} \mathrm{W}$, on soil, CB \& BF, 2 August 1979.

* Cladonia macroceras (Delise) Hav. - N. E. Balle $\varnothing, 75^{\circ} 12^{\prime} \mathrm{N}, 58^{\circ} 54^{\prime} \mathrm{W}$, on soil, BF, 14 July 1979; Tugtuligssuaq, $75^{\circ} 24^{\prime} \mathrm{N}, 58^{\circ} 33^{\prime} \mathrm{W}$, on soil, CB \& BF, 22 July 1979; Tugtuligssuaq, southwest of point $863,75^{\circ} 26^{\prime} \mathrm{N}, 58^{\circ} 12^{\prime} \mathrm{W}$, alt. $720 \mathrm{~m}$, on soil, $\mathrm{CB}$ \& BF, 7 August 1979.

Cladonia pyxidata (L.) Hoffm. - N. E. Balle Ø, $75^{\circ} 12^{\prime} \mathrm{N}, 58^{\circ} 35^{\prime} \mathrm{W}$, on soil, BF, 14 July 1979 ; Tugtuligssuaq, Igdluminerssuit, $75^{\circ} 22^{\prime} \mathrm{N}, 58^{\circ} 32^{\prime} \mathrm{W}$, alt. 25 m, on soil, CB \& BF, 25 July 1979; Tugtuligssuaq, point $619,75^{\circ} 24^{\prime} \mathrm{N}, 58^{\circ} 31^{\prime} \mathrm{W}$, alt. $619 \mathrm{~m}$, on soil, CB \& BF, 22 July 1979; Tugtuligssuaq, $75^{\circ} 24^{\prime} \mathrm{N}$, $58^{\circ} 33^{\prime} \mathrm{W}$, alt $350 \mathrm{~m}$, on soil in fell field, CB \& BF, 22 July 1979.

* Cladonia rangiferina (L.) F. H. Wigg. - Tugtuligssuaq, c. $75^{\circ} 23^{\prime} \mathrm{N}, 58^{\circ} 31^{\prime} \mathrm{W}$, on soil, $\mathrm{CB} \& \mathrm{BF}, 2$ August 1979.

* Cladonia sulphurina (Michx.) Fr. - Tugtuligssuaq, c. $75^{\circ} 23^{\prime} \mathrm{N}, 58^{\circ} 31^{\prime} \mathrm{W}$, on soil, $\mathrm{CB} \& \mathrm{BF}, 2$ August 1979.

* Cladonia trassii Ahti - N. E. Balle Ø, $75^{\circ} 12^{\prime} \mathrm{N}$, $58^{\circ} 54^{\prime} \mathrm{W}$, on soil rich in humus, BF, 14 July 1979 ; Tugtuligssuaq, c. $75^{\circ} 23^{\prime} \mathrm{N}, 58^{\circ} 31^{\prime} \mathrm{W}$, on soil, CB \& BF, 2 August 1979.

* Dactylina arctica (Hook.) Nyl. - Tugtuligssuaq, 
area south of point $419,75^{\circ} 22^{\prime} \mathrm{N}, 58^{\circ} 35^{\prime} \mathrm{W}$, alt. $250 \mathrm{~m}$, on Racomitrium lanuginosum tussock on soil, $\mathrm{CB} \&$ BF, 18 July 1979 ; Tugtuligssuaq, point $619,75^{\circ} 24^{\prime} \mathrm{N}$, $58^{\circ} 31^{\prime} \mathrm{W}$, alt. $619 \mathrm{~m}$, on Racomitrium lanuginosum tussock together with Gowardia nigricans, CB \& BF, 22 July 1979; Tugtuligssuaq, Itivdlip aluk, $75^{\circ} 25^{\prime} \mathrm{N}$, $58^{\circ} 21^{\prime} \mathrm{W}$, alt. $80 \mathrm{~m}$, on moss tussock together with Rinodina archaea, CB \& BF, 8 August 1979.

* Dactylina ramulosa (Hook.) Tuck. - Tugtuligssuaq, area east of point $619,75^{\circ} 25^{\prime} \mathrm{N}, 58^{\circ} 27^{\prime} \mathrm{W}$, alt. $600 \mathrm{~m}$, on soil together with Gowardia nigricans, CB \& BF, 29 July 1979; Tugtuligssuaq, area southwest of point $863,75^{\circ} 26^{\prime} \mathrm{N}, 58^{\circ} 12^{\prime} \mathrm{W}$, alt. $720 \mathrm{~m}$, on moist soil, CB \& BF, 7 August 1979.

Flavocetraria cucullata (Bellardi) Kärnefelt \& Thell - Tugtuligssuaq, Igdluminerssuit, $75^{\circ} 22^{\prime} \mathrm{N}$, $58^{\circ} 35^{\prime} \mathrm{W}$, alt. $25 \mathrm{~m}$, on soil together with Bryocaulon divergens, Cladonia amaurocraea and Thamnolia vermicularis v. subuliformis, CB \& BF, 25 July 1979.

* Flavocetraria nivalis (L.) Kärnefelt \& Thell Summit of Red Head, $75^{\circ} 04^{\prime} \mathrm{N}, 58^{\circ} 06^{\prime} \mathrm{W}$, alt. c. $250 \mathrm{~m}$, on soil rich in humus, BF, 14 July 1979; Depot Øer, the easternmost island, $75^{\circ} 07^{\prime} \mathrm{N}, 58^{\circ} 33^{\prime} \mathrm{W}$, on soil, BF, 14 July 1979; N. E. Balle Ø, $75^{\circ} 12^{\prime}$ N, $58^{\circ} 54^{\prime} \mathrm{W}$, on soil, BF, 14 July 1979; Tugtuligssuaq, Igdluminerssuit, $75^{\circ} 22^{\prime} \mathrm{N}, 58^{\circ} 37^{\prime} \mathrm{W}$, alt. $25 \mathrm{~m}$, on soil, CB \& BF, 23 July 1979; Tugtuligssuaq, 75²4'N, $58^{\circ} 33^{\prime} \mathrm{W}$, alt. $350 \mathrm{~m}$, on soil, $\mathrm{CB} \& \mathrm{BF}, 2$ August 1979; Tugtuligssuaq, area between Itivdlip aluk and point $480,75^{\circ} 26^{\prime} \mathrm{N}, 58^{\circ} 22^{\prime} \mathrm{W}$, alt. $350 \mathrm{~m}$, on soil, $\mathrm{CB}$ $\&$ BF, 8 August 1979; Sabine Øer, the biggest island, $75^{\circ} 30^{\prime} \mathrm{N}, 60^{\circ} 13^{\prime} \mathrm{W}$, on soil, BF, 14 July 1979.

* Gowardia nigricans (Ach.) P. Halonen, L. Myllus, S. Velmela \& H. Hyvärinen - N. E. Balle $\varnothing, 75^{\circ} 12^{\prime} \mathrm{N}, 58^{\circ} 54^{\prime} \mathrm{W}$, on Racomitrium lanuginosum tussock, BF, 14 July 1979; Tugtuligssuaq, point $619,75^{\circ} 24^{\prime} \mathrm{N}, 58^{\circ} 31^{\prime} \mathrm{W}$, alt. $619 \mathrm{~m}$, on Racomitrium lanuginosum tussock, CB \& BF, 22 July 1979; Tugtuligssuaq, $75^{\circ} 24^{\prime} \mathrm{N}, 58^{\circ} 33^{\prime} \mathrm{W}$, alt. $350 \mathrm{~m}$, on soil, CB \& BF, 22 July 1979; Tugtuligssuaq, area east of point $619,75^{\circ} 25^{\prime} \mathrm{N}, 58^{\circ} 27^{\prime} \mathrm{W}$, alt. $600 \mathrm{~m}$, on soil, $\mathrm{CB}$ \& BF, 29 July 1997; Sabine Øer, the biggest island, $75^{\circ} 30^{\prime} \mathrm{N}, 60^{\circ} 13^{\prime} \mathrm{W}$, on soil, BF, 14 July 1979.

* Lecanora hagenii (Ach.) Ach. v. fallax Hepp Tugtuligssuaq, c. $75^{\circ} 23^{\prime} \mathrm{N}, 58^{\circ} 31^{\prime} \mathrm{W}$, on old bone, CB \& BF, 2 August 1979.

Melanelia hepatizon (Ach.) Thell - Tugtuligssuaq, Igdluminerssuit, $75^{\circ} 22^{\prime} \mathrm{N}, 58^{\circ} 32^{\prime} \mathrm{W}$, alt.
20 m, on siliceous stone, CB \& BF, 24 July 1979.

* Ochrolechia frigida (Sw.) Lynge - Depot Øer, the easternmost island, $75^{\circ} 07^{\prime} \mathrm{N}, 58^{\circ} 33^{\prime} \mathrm{W}$, on soil, BF, 14 July 1979; Tugtuligssuaq, c. $75^{\circ} 23^{\prime} \mathrm{N}$, $58^{\circ} 31^{\prime} \mathrm{W}$, on soil, CB \& BF, 2 August 1979.

* Ochrolechia grimmiae Lynge - Tugtuligssuaq, east of point $619,75^{\circ} 25^{\prime} \mathrm{N}, 58^{\circ} 27^{\prime} \mathrm{W}$, alt. $600 \mathrm{~m}$, on mosses, CB \& BF, 29 July 1979.

* Ophioparma lapponica (Räsänen) Hafellner \& R. W. Rogers - Tugtuligssuaq, Igdluminerssuit, $75^{\circ} 22^{\prime} \mathrm{N}, 58^{\circ} 32^{\prime} \mathrm{W}$, alt. $20 \mathrm{~m}$, on siliceous stone, $\mathrm{CB}$ \& BF, 24 July 1979; Tugtuligssuaq, Kap Seddon, $75^{\circ} 22^{\prime} \mathrm{N}, 58^{\circ} 39^{\prime} \mathrm{W}$, alt. $300 \mathrm{~m}$, on gneissic boulder on talus slope together with Orphniospora moriopsis, Pseudephebe minuscula, Rhizocarpon inarense and Umbilicaria hyperborea, CB \& BF, 20 July 1979.

* Orphniospora moriopsis (A. Massal.) D. Hawksw. - Tugtuligssuaq, Kap Seddon, $75^{\circ} 22^{\prime} \mathrm{N}$, $58^{\circ} 39^{\prime} \mathrm{W}$, alt. $300 \mathrm{~m}$, on siliceous rock, CB \& BF, 20 July 1979.

* Parmelia saxatilis (L.) Ach. - Sabine Øer, the biggest island, $75^{\circ} 30^{\prime} \mathrm{N}, 60^{\circ} 13^{\prime} \mathrm{W}$, on rock, $\mathrm{BF}, 14$ July 1979.

* Parmelia skultii Hale - Tugtuligssuaq, area southwest of point $863,75^{\circ} 26^{\prime} \mathrm{N}, 58^{\circ} 12^{\prime} \mathrm{W}$, alt. 720 m, on soil among mosses, CB \& BF, 7 August 1979.

* Peltigera didactyla (With.) J. R. Laundon Tugtuligssuaq, Kap Seddon, $75^{\circ} 22^{\prime} \mathrm{N}, 58^{\circ} 38^{\prime} \mathrm{W}$, alt. 25 m, on soil, CB \& BF, 2 August 1979.

* Peltigera malacea (Ach.) Funck - Depot Øer, the easternmost island, $75^{\circ} 07^{\prime} \mathrm{N}, 58^{\circ} 33^{\prime} \mathrm{W}$, on soil, BF, 14 July 1979.

* Peltigera rufescens (Weiss) Humb. - Tugtuligssuaq, Igdluminerssuit, $75^{\circ} 22^{\prime} \mathrm{N}, 58^{\circ} 32^{\prime} \mathrm{W}$, alt. 25 m, on soil, CB \& BF, 25 July 1979.

* Pertusaria oculata (Dicks.) Th. Fr. - N. E. Balle $\varnothing, 75^{\circ} 12^{\prime} \mathrm{N}, 58^{\circ} 54^{\prime} \mathrm{W}$, on soil, BF, 14 July 1979; Tugtuligssuaq, area east of Igdluminerssuit, $75^{\circ} 22^{\prime} \mathrm{N}, 58^{\circ} 32^{\prime} \mathrm{W}$, alt. $10 \mathrm{~m}$, on plant remains, $\mathrm{CB}$ \& BF, 31 July 1979; Tugtuligssuaq, c. $75^{\circ} 23^{\prime} \mathrm{N}$, $58^{\circ} 31^{\prime} \mathrm{W}$, on soil, CB \& BF, 2 August 1979.

* Pleopsidium chlorophanum (Wahlenb.) Zopf Tugtuligssuaq, point $619,75^{\circ} 24^{\prime} \mathrm{N}, 58^{\circ} 31^{\prime} \mathrm{W}$, alt. 619 m, on gneissic rock, CB \& BF, 22 July 1979.

* Pseudephebe minuscula (Nyl. ex Arnold) Brodo \& D. Hawksw. - Tugtuligssuaq, Igdluminerssuit, $75^{\circ} 22^{\prime} \mathrm{N}, 58^{\circ} 32^{\prime} \mathrm{W}$, alt. $20 \mathrm{~m}$, on siliceous stone, $\mathrm{CB}$ \& BF, 24 July 1979; Tugtuligssuaq, Kap Seddon, 
$75^{\circ} 22^{\prime} \mathrm{N}, 58^{\circ} 39^{\prime} \mathrm{W}$, alt. $300 \mathrm{~m}$, on gneissic boulder, CB \& BF, 20 July 1979; Sabine Øer, the biggest island, $75^{\circ} 30^{\prime} \mathrm{N}, 60^{\circ} 13^{\prime} \mathrm{W}$, on Sphaerophorus fragilis, BF, 14 July 1979.

* Pseudephebe pubescens (L.) M. Choisy - Tugtuligssuaq, Igdluminerssuit, $75^{\circ} 22^{\prime} \mathrm{N}, 58^{\circ} 32^{\prime} \mathrm{W}$, alt. $5 \mathrm{~m}$, on rock, CB \& BF, 20 July 1979; Tugtuligssuaq, $75^{\circ} 24^{\prime} \mathrm{N}, 58^{\circ} 33^{\prime} \mathrm{W}$, alt. $350 \mathrm{~m}$, on rock, CB \& BF, 22 July 1979 .

* Psoroma tenue Henssen v. boreale Henssen Tugtuligssuaq, Igdluminerssuit, $75^{\circ} 22^{\prime} \mathrm{N}, 58^{\circ} 37^{\prime} \mathrm{W}$, alt. $10 \mathrm{~m}$, on soil rich in humus, CB \& BF, 28 July 1979 ; Tugtuligssuaq, c. $75^{\circ} 23^{\prime} \mathrm{N}, 58^{\circ} 31^{\prime} \mathrm{W}$, on plant remains, CB \& BF, 2 August 1979.

* Rhizocarpon inarense (Vain.) Vain. - Tugtuligssuaq, Kap Seddon, $75^{\circ} 22^{\prime} \mathrm{N}, 58^{\circ} 39^{\prime} \mathrm{W}$, alt. 300 m, on gneissic boulder, CB \& BF, 20 July 1979.

* Rinodina archaea (Ach.) Arnold - Tugtuligssuaq, Itivdlip aluk, $75^{\circ} 25^{\prime} \mathrm{N}, 58^{\circ} 21^{\prime} \mathrm{W}$, alt. $80 \mathrm{~m}$, on moss hummock, CB \& BF, 8 August 1979.

* Rinodina mniaraea (Ach.) Körb. - Tugtuligssuaq, $75^{\circ} 24^{\prime} \mathrm{N}, 58^{\circ} 33^{\prime} \mathrm{W}$, alt. $350 \mathrm{~m}$, on mosses, CB \& BF, 22 July 1979.

* Rinodina turfacea (Wahlenb.) Körb. - N. E. Balle $\varnothing, 75^{\circ} 12^{\prime} \mathrm{N}, 58^{\circ} 54^{\prime} \mathrm{W}$, on soil rich in humus, BF, 14 July 1979.

* Solorina bispora Nyl. - Tugtuligssuaq, area east of Itivdlip aluk, $75^{\circ} 25^{\prime} \mathrm{N}, 58^{\circ} 18^{\prime} \mathrm{W}$, alt. $25 \mathrm{~m}$, on soil on SW rock, CB \& BF, 8 August 1979.

* Solorina crocea (L.) Ach. - Tugtuligssuaq, point $619,75^{\circ} 24^{\prime} \mathrm{N}, 58^{\circ} 31^{\prime} \mathrm{W}$, alt. $619 \mathrm{~m}$, on soil, CB \& BF, 22 July 1979; Tugtuligssuaq, area southwest of point $863,75^{\circ} 26^{\prime} \mathrm{N}, 58^{\circ} 12^{\prime} \mathrm{W}$, alt. $720 \mathrm{~m}$, on moist soil, CB \& BF, 7 August 1979.

* Sphaerophorus fragilis (L.) Pers. - N. E. Balle $\varnothing, 75^{\circ} 12^{\prime} \mathrm{N}, 58^{\circ} 54^{\prime} \mathrm{W}$, on soil, BF, 14 July 1979 ; Tugtuligssuaq, point $619,75^{\circ} 24^{\prime} \mathrm{N}, 58^{\circ} 31^{\prime} \mathrm{W}$, alt. 619 m, on soil, CB \& BF, 22 July 1979; Tugtuligssuaq, point $863,75^{\circ} 27^{\prime} \mathrm{N}, 58^{\circ} 11^{\prime} \mathrm{W}$, alt. $860 \mathrm{~m}$, on soil, BF, 7 August 1979; Sabine Øer, the biggest island, $75^{\circ} 30^{\prime} \mathrm{N}, 60^{\circ} 13^{\prime} \mathrm{W}$, on soil, BF, 14 July 1979.

* Sphaerophorus globosus (Huds.) Vain. - N. E. Balle $\varnothing, 75^{\circ} 12^{\prime} \mathrm{N}, 58^{\circ} 54^{\prime} \mathrm{W}$, on soil rich in humus, BF, 14 July 1979; Tugtuligssuaq, $75^{\circ} 24^{\prime} \mathrm{N}, 58^{\circ} 31^{\prime} \mathrm{W}$, alt. $525 \mathrm{~m}$, on soil in east-facing snow patch with $\mathrm{Sa}$ lix herbacea, CB \& BF, 2 August 1979.

* Stereocaulon alpinum Laurer - Sabine Øer, the biggest island, $75^{\circ} 30^{\prime} \mathrm{N}, 60^{\circ} 13^{\prime} \mathrm{W}$, on soil, BF, 14
July 1979

* Stereocaulon botryosum Ach. - Tugtuligssuaq, c. $75^{\circ} 23^{\prime} \mathrm{N} .58^{\circ} 31^{\prime} \mathrm{W}$, on rock, CB \& BF, 2 August 1979.

* Stereocaulon glareosum (Savicz) H. Magn. - N. E. Balle $\varnothing, 75^{\circ} 12^{\prime} \mathrm{N}, 58^{\circ} 54^{\prime} \mathrm{W}$, on soil, BF, 14 July 1979; Tugtuligssuaq, point $619,75^{\circ} 24^{\prime} \mathrm{N}, 58^{\circ} 31^{\prime} \mathrm{W}$, alt. 619 m, on soil, CB \& BF, 22 July 1979; Tugtuligssuaq, point $863,75^{\circ} 27^{\prime} \mathrm{N}, 58^{\circ} 11^{\prime} \mathrm{W}$, alt. $860 \mathrm{~m}$, BF, 7 August 1979.

* Thamnolia vermicularis (Sw.) Schaer. v. subuliformis (Ehrh.) Schaer. - Summit of Red Head, $75^{\circ} 04^{\prime} \mathrm{N}, 58^{\circ} 06^{\prime} \mathrm{W}$, alt. $260 \mathrm{~m}$, on soil, BF, 14 July $1979 ;$ N. E. Balle $\varnothing, 75^{\circ} 12^{\prime} \mathrm{N}, 58^{\circ} 54^{\prime} \mathrm{W}$, on Racomitrium lanuginosum tussock, BF, 14 July 1979; Tugtuligssuaq, Igdluminerssuit, $75^{\circ} 22^{\prime} \mathrm{N}, 58^{\circ} 35^{\prime} \mathrm{W}$, alt. $25 \mathrm{~m}$, on soil, CB \& BF, 25 July 1979.

Umbilicaria arctica (Ach.) Nyl. - N. E. Balle Ø, $75^{\circ} 12^{\prime} \mathrm{N}, 58^{\circ} 54^{\prime} \mathrm{W}$, on rock, BF, 14 July 1979 ; Sabine Øer, the biggest island, $75^{\circ} 30^{\prime} \mathrm{N}, 60^{\circ} 13^{\prime} \mathrm{W}$, on rock, BF, 14 July 1979.

* Umbilicaria hyperborea (Ach.) Hoffm. - Tugtuligssuaq, Kap Seddon, $75^{\circ} 22^{\prime} \mathrm{N}, 58^{\circ} 39^{\prime} \mathrm{W}$, alt. 300 m, on rock, CB \& BF, 20 July 1979.

* Umbilicaria virginis Schaer. - N. E. Balle Ø, $75^{\circ} 12^{\prime} \mathrm{N}, 58^{\circ} 54^{\prime} \mathrm{W}$, on rock, BF, 14 July 1979 ; Tugtuligssuaq, $75^{\circ} 24^{\prime} \mathrm{N}, 58^{\circ} 31^{\prime} \mathrm{W}$, alt. $590 \mathrm{~m}$, on SE rock, CB \& BF, 22 July 1979; Tugtuligssuaq, point $619,75^{\circ} 24^{\prime} \mathrm{N}, 58^{\circ} 31^{\prime} \mathrm{W}$, alt. $619 \mathrm{~m}$, on rock, CB \& BF, 22 July 1979.

Until recently Melville Bugt has represented the greatest hiatus as regards the range of lichen vegetation along the west coast of Greenland, and it still does to some extent, as the knowledge about the saxicolous lichens and the microlichens in general is very incomplete. As regards the terricolous vegetation, snow patch communities appear to be of great importance on the Tugtuligssuaq peninsula. A snow patch with Arctocetraria nigricascens, Dactylina ramulosa, Parmelia skultii and Solorina crocea is comparable with a Cassiope snow patch heath on the terraces above Qaanaaq in the Thule district previously described by the author (Hansen, 1989, 2011). It also contains the rare species Cladonia alaskana A. Evans, which, however, was not found by C. Bay and B. Fredskild. Parmelia skultii is a rare Greenland lichen also known from the north coast of Greenland (HANSEN, 2009). The more or less fragmen- 
tary dwarf shrub heaths and fell fields on Tugtuligssuaq and the islands are fairly rich in macrolichens such as Alectoria ochroleuca, Bryocaulon divergens, Bryoria nitidula, Cetraria islandica, Cladonia macroceras, Flavocetraria cucullata, F. nivalis, Sphaerophorus fragilis, S. globosus and Thamnolia vermicularis v. subuliformis. Cetrariella delisei and Cladonia trassii grow in moist depressions in the heaths. Solorina bispora is restricted to base-rich soil probably near a basaltic dyke or intrusion. A number of lichens such as Caloplaca nivalis, Dactylina arctica, Gowardia nigricans and Ochrolechia grimmiae have a distinct preference for moss tussocks. Bryoria chalybeiformis occurs commonly on talus slopes. Saxicolous lichens Brodoa oroarctica and Ophioparma lapponica with a preference for rock faces exposed to strong winds. These lichens are fairly well represented in C. Bay's and B. Fredskild's collections, which otherwise contain some common, widely distributed species such as Melanelia hepatizon, Orphniospora moriopsis, Pseudephebe minuscula, P. pubescens, Rhizocarpon inarense, Umbilicaria hyperborea and $U$. virginis. Apart from Umbilicaria arctica nitrophilous lichens apparently have been neglected by the two collectors. Candelariella aurella and Lecanora hagenii v. fallax grow on old bone as it is often seen in Greenland, in particular near former Eskimo settlements. Future collecting work along Melville Bugt should concentrate on saxicolous lichens, in particular microlichens.

\section{ACKNOWLEDGEMENTS}

I wish to thank Christian Bay and Bent Fredskild for handing over their lichen collections to me for identification and verification.

\section{REFERENCES}

Fredskild B., Hanfgarn S., Feilberg J., Alstrup V., 1979: Grønlands Botaniske Undersøgelse 1978 og 1979. Teknisk Rapport. - København.

Hansen E. S., 1981: Cetraria nigricascens and $C$. tilesii found in Greenland. - Lichenologist, 13: 97-99.

HAnsen E. S., 1989: The lichen flora of Qaanaaq (Thule), northwestern Greenland. - Mycotaxon, 35: 379-394.

Hansen E. S., 1995: Greenland Lichens. - Copenhagen.

HANSEn E. S., 2007: Lichens from Upernavik island, NW Greenland. - Willdenowia, 37: 353-362.

HANSEN E. S., 2009: Lichens from Johannes V. Jensen Land, N Greenland, the northernmost arctic land area. - Willdenowia, 39: 179-186.

Hansen E. S., 2011: Lichens from Qaanaaq and Siorapaluk, North West Greenland. - Folia Cryptogamica Estonica, 48: 5-11.

Jensen B. (ed.), 1999: Grønlands Biodiversitet - et landestudie. Teknisk Rapport 27. - Nuuk.

Kristinsson H., Zhurbenko M., Hansen E. S., 2010: Panarctic checklist of lichens and lichenicolous fungi. Technical Report, 20. - Akureyri.

Santesson R., Moberg R., Nordin A., Tønsberg T., VITIKAINEN O., 2004: Lichen-forming and lichenicolous fungi of Fennoscandia. - Uppsala.

Sørensen T., 1943: The flora of Melville Bugt. Meddelelser om Grønland, 124: 1-70.

Thomson J. W., 1984: American Arctic lichens. I. The macrolichens. - New York.

Thomson J. W., 1997: American Arctic lichens. II. The microlichens. - Wisconsin.

Øvstedal D., Tønsberg T., Elvebakk A., 2009: The lichen flora of Svalbard. - Sommerfeltia, 33: $1-393$.

\section{PAPILDOMI DUOMENYS APIE MELVILLE BUGT (ŠIAURĖS VAKARINE் GRENLANDIJA) KERPIU FLORĄ ŠIAURIAU $75^{\circ} \mathrm{N}$ PLATUMOS}

\section{Eric Steen Hansen}

\section{Santrauka}

Pateikiamas 59 kerpių rūšių sąrašas iš Melville Bugt vietovès šiauriau $75^{\circ} \mathrm{N}$ platumos. Dauguma ju (54) aptiktos šioje vietovejje pirmą kartą. Dauguma kerpių rūšiu aptiktos ant uolų ir dirvožemio daugiau ar mažiau vejo veikiamuose tundros šlaituose bei ploteliuose su ilgiau išsilaikančiu sniegu. 